\title{
Suplementação de colina em dietas para frangos de corte machos na fase inicial de criação
}

\author{
[Levels of supplementation of choline in diets for male broilers in initial phase] \\ M.A. Pompeu ${ }^{1}$, L.J.C. Lara $^{2}$, N.C. Baião ${ }^{2}$, R. Ecco ${ }^{2}$, S.V. Cançado ${ }^{2}$, \\ J.S.R. Rocha ${ }^{1}$, A.L.C. Machado ${ }^{1}$, R.J.C. Vasconcelos ${ }^{1}$ \\ ${ }^{1}$ Aluno de pós-graduação - Escola de Veterinária - UFMG, Belo Horizonte, MG \\ ${ }^{2}$ Escola de Veterinária - UFMG \\ Caixa Postal 527 \\ 30123-970 - Belo Horizonte, MG
}

\begin{abstract}
RESUMO
Foram avaliados os efeitos da suplementação de colina em dietas para frangos de corte sobre o desempenho produtivo e a composição da carcaça e do fígado e sobre as características macroscópicas e histopatólogicas do fígado na fase inicial de criação. Os tratamentos foram definidos pela suplementação de colina - zero, 100, 200, 300 e 400mg de colina/kg de ração, durante o período de criação de um a 21 dias de idade. As dietas experimentais, formuladas à base de milho, farelo de soja e farinha de carne, foram isonutritivas, com exceção dos níveis de colina. O delineamento experimental utilizado foi o inteiramente ao acaso, e a estimativa da suplementação de colina foi avaliada pelos modelos de regressão. A suplementação de colina apresentou efeito linear significativo para conversão alimentar, e as demais características avaliadas não foram influenciadas pela suplementação. A suplementação de 400mg colina/kg de ração melhora a conversão alimentar de frangos de corte aos 21 dias de idade.
\end{abstract}

Palavras-chave: frango de corte, colina, desempenho, fígado

\begin{abstract}
The effects of supplemental levels of choline on performance, carcass composition and gross and histopatology characteristics of the livers in the initial phase in diets for broiler chickens were evaluated. The treatments were defined by the levels of choline supplementation (zero, 100, 200, 300 and 400mg choline/kg diet). The period of creation was one to 21 days-old. Diets formulated based on corn, soybean meal and meat meal are constituted by isonutrient except the levels of choline. The experimental design was completely randomized and the estimation of choline supplementation was evaluated by regression models. The levels of choline supplementation showed a significant linear effect for the $F$ test $(P \leq 0.05)$. Values for feed and other parameters were not influenced by supplementation $(P>0.05)$. The supplementation of $400 \mathrm{mg}$ choline/kg diet improves feed conversion of broilers at 21 days old.
\end{abstract}

Keywords: broiler, choline, performance, liver

\section{INTRODUÇÃO}

A colina é considerada um nutriente essencial ao organismo animal. Sua classificação é controversa, pois não se enquadra na classificação clássica das vitaminas e, em função disso, talvez essa designação não seja a correta. Alguns nutricionistas não consideram a colina como uma vitamina do complexo B pois esta não

Recebido em 1 de setembro de 2010

Aceito em 5 de outubro de 2011

E-mail:marianapompeu@hotmail.com participa no metabolismo como coenzima. A colina serve para a síntese de lecitina e de outros fosfolipídios que participam da estrutura das células e, consequentemente, dos tecidos. Além disto, é exigida em quantidades muito superiores às outras vitaminas do grupo B (Bertechini, 2006).

Do ponto de vista bioquímico, a colina tem participação nas sínteses de lecitina, 
esfingomielina e acetilcolina. A lecitina participa na absorção e no transporte das gorduras ao fígado e posterior mobilização e transporte das gorduras hepáticas. Além disso, faz parte da composição das membranas celulares e organelas como mitocôndrias e microssomas. A esfingomielina é um fosfolipídio contendo um ácido graxo, um radical fosfórico, colina e um álcool aminado. A acetilcolina é a substância mediadora da atividade nervosa, produzida a partir da colina e do ácido acético, sendo responsável pela transmissão dos estímulos nervosos (Ferreira e Neto, 2003).

A colina é encontrada praticamente em todos os ingredientes utilizados na formulação de dietas de aves. No entanto, as fontes proteicas de origem animal são as mais ricas nesta vitamina. O milho e o farelo de soja apresentam 1.000 e $2.700 \mathrm{mg} / \mathrm{kg}$ de colina, respectivamente, e sua biodisponibilidade varia de 60 a $75 \%$. As farinhas de origem animal apresentam colina com biodisponibilidade acima de $90 \%$. As farinhas de carne e ossos e de peixe contêm 1.950 e $3.500 \mathrm{mg} / \mathrm{kg}$ de colina, respectivamente (Bertechini, 2006).

As aves em crescimento têm uma determinada exigência de colina que não pode ser substituída por altos níveis de metionina ou por outros doadores de grupos metil na dieta (Jukes, 1940; Jukes, 1941). Sua deficiência é prontamente percebida em frangos alimentados com dieta pobre em colina, ou em seus precursores. A facilidade de se produzir deficiência em frangos sugere que a biossíntese desta seja menos eficiente nos frangos quando comparada com as aves de postura (Nesheim et al., 1971).

O objetivo deste trabalho foi avaliar os efeitos da suplementação de colina em dietas para frangos de corte no período de um a 21 dias de idade sobre o desempenho produtivo e a composição da carcaça e do fígado e sobre as características macroscópicas e histopatológicas do fígado.

\section{MATERIAL E MÉTODOS}

As aves foram alojadas em galpão convencional, dividido em boxes idênticos com $2,5 \mathrm{~m}^{2}$ cada, sendo 30 boxes de cada lado. O material utilizado como cama foi o cepilho de madeira. As aves foram aquecidas nos primeiros 14 dias com uma lâmpada infravermelha (250Watts) colocada em cada boxe. Nos primeiros sete dias de alojamento, foi utilizado um bebedouro tipo copo de pressão e, após esse período, um bebedouro pendular para cada boxe. Foram utilizados dois comedouros tipo tubular infantil até os 14 dias de idade, posteriormente substituídos por um comedouro do tipo tubular adulto para cada boxe.

Foram utilizados 1.080 pintos de corte, machos, da linhagem Cobb, de um dia de idade, sendo alojados 36 por boxe $\left(14,4\right.$ aves $\left./ \mathrm{m}^{2}\right)$. Os pintos foram vacinados, no incubatório de origem, contra doença de Marek e, aos 14 dias de idade, contra doença de Gumboro, via água de bebida. Água e ração foram oferecidas à vontade. O período de criação das aves foi de um a 21 dias de idade.

Para a formulação e o cálculo nutricionais, foram considerados os valores dos ingredientes estabelecidos nas tabelas brasileiras sobre exigências de aves e suínos (Rostagno et al., 2005). Os valores nutricionais foram utilizados de acordo com Leite et al. (2009). As dietas foram isonutritivas, com exceção da quantidade de colina. O suplemento de colina utilizado cloreto de colina - foi analisado pelo método de Reinecke Salt (Glick, 1944). A média dos resultados das amostras analisadas foi de $60,8 \%$, o que comprova que o suplemento utilizado encontrava-se dentro dos padrões esperados. As rações experimentais foram peletizadas $\mathrm{e}$ trituradas.

A composição percentual das dietas experimentais e os valores nutricionais calculados encontram-se na Tab. 1.

Os tratamentos foram definidos pela suplementação de colina nas dietas experimentais, formuladas para atender às exigências nutricionais, exceto quanto ao valor de colina, da seguinte forma: zero, 100, 200, 300, $400 \mathrm{mg} / \mathrm{kg}$. 
Tabela 1. Composição percentual das dietas da fase inicial e seus respectivos valores nutricionais calculados de acordo com a suplementação de colina $(\mathrm{mg} / \mathrm{kg})$

\begin{tabular}{|c|c|c|c|c|c|}
\hline Ingrediente & Zero & 100 & 200 & 300 & 400 \\
\hline Milho grão & 59,75 & 59,73 & 59,71 & 59,70 & 59,68 \\
\hline Gordura de aves & 0,975 & 0,975 & 0,975 & 0,975 & 0,975 \\
\hline Farelo de soja $46 \%$ PB & 33,80 & 33,80 & 33,80 & 33,80 & 33,80 \\
\hline Farinha de carne & 3,925 & 3,925 & 3,925 & 3,925 & 3,925 \\
\hline Sal comum & 0,221 & 0,221 & 0,221 & 0,221 & 0,221 \\
\hline Calcário 38\% & 0,787 & 0,787 & 0,787 & 0,787 & 0,787 \\
\hline Supl. vitamínico ${ }^{1}$ & 0,035 & 0,035 & 0,035 & 0,035 & 0,035 \\
\hline Supl. mineral ${ }^{1}$ & 0,050 & 0,050 & 0,050 & 0,050 & 0,050 \\
\hline Bicarbonato de $\mathrm{Na}$ & 0,150 & 0,150 & 0,150 & 0,150 & 0,150 \\
\hline Fitase* & 0,015 & 0,015 & 0,015 & 0,015 & 0,015 \\
\hline DL-metionina $98 \%$ & 0,180 & 0,180 & 0,180 & 0,180 & 0,180 \\
\hline L-lisina $80 \%$ & 0,046 & 0,046 & 0,046 & 0,046 & 0,046 \\
\hline Treonina 98\% & 0,010 & 0,010 & 0,010 & 0,010 & 0,010 \\
\hline Cloreto colina $60 \%$ & - & 0,016 & 0,033 & 0,050 & 0,067 \\
\hline Avilamicina 20\% & 0,005 & 0,005 & 0,005 & 0,005 & 0,005 \\
\hline Salinomicina $12 \%$ & 0,050 & 0,050 & 0,050 & 0,050 & 0,050 \\
\hline Nicarbazina 98\% & 0,004 & 0,004 & 0,004 & 0,004 & 0,004 \\
\hline Total (\%) & 100,0 & 100,0 & 100,0 & 100,0 & 100,0 \\
\hline \multicolumn{6}{|l|}{ Valor nutricional } \\
\hline Proteína bruta (\%) & 22,30 & 22,30 & 22,30 & 22,30 & 22,30 \\
\hline Energia metabolizável (kcal/kg) & 2.930 & 2.930 & 2.930 & 2.930 & 2.930 \\
\hline Cálcio (\%) & 0,990 & 0,990 & 0,990 & 0,990 & 0,990 \\
\hline Fósforo disponível (\%) & 0,500 & 0,500 & 0,500 & 0,500 & 0,500 \\
\hline Lisina digestível (\%) & 1,100 & 1,100 & 1,100 & 1,100 & 1,100 \\
\hline Metionina digestível (\%) & 0,470 & 0,470 & 0,470 & 0,470 & 0,470 \\
\hline Met + Cis digestível (\%) & 0,760 & 0,760 & 0,760 & 0,760 & 0,760 \\
\hline Treonina digestível (\%) & 0,710 & 0,710 & 0,710 & 0,710 & 0,710 \\
\hline Sódio (\%) & 0,200 & 0,200 & 0,200 & 0,200 & 0,200 \\
\hline Colina total $(\mathrm{mg} / \mathrm{kg})$ & 1.367 & 1.467 & 1.567 & 1.667 & 1.767 \\
\hline
\end{tabular}

${ }^{1}$ Suplementação de vitaminas e minerais (quantidades por kg do produto): vit. A 9.000.000UI; vit. D3 2.500.000UI; vit. E 20.000UI; vit. $\mathrm{K}_{3} 2.500 \mathrm{mg}$; vit. $\mathrm{B}_{1} 2.000 \mathrm{mg}$; vit. $\mathrm{B}_{2} 5.000 \mathrm{mg}$; vit. $\mathrm{B}_{6} 2.500 \mathrm{mg}$; vit. $\mathrm{B}_{12} 14.000 \mathrm{mcg}$; biotina $80 \mathrm{mg}$; niacina $35.000 \mathrm{mg}$; ácido fólico $1.000 \mathrm{mg}$; ácido pantotênico $12.000 \mathrm{mg}$; $\mathrm{Mn} 90.000 \mathrm{mg}$; Zn $80.000 \mathrm{mg}$; $\mathrm{Fe}$ 30.000mg; Cu 10.000mg; Se 290mg. *Ronozyme ${ }^{\circledR}$

As variáveis estudadas foram: consumo de ração, ganho de peso, conversão alimentar, taxa de viabilidade, composição de carcaça - matéria seca, matéria mineral, proteína bruta e extrato etéreo - composição de extrato etéreo, avaliação macroscópica e histopatológica do fígado das aves. A carcaça inteira, sem cabeça e pés, e o fígado foram pesados e embalados em sacos plásticos identificados. Foram armazenados em câmara de congelamento a $-18^{\circ} \mathrm{C}$. Cada carcaça inteira foi moída individualmente em moedor de carne convencional. Foi realizada a pré-secagem das amostras de carcaça em estufa de ventilação forçada por 72 horas a $65^{\circ} \mathrm{C}$ (Silva e Queiroz, 2002). Após a pré-secagem, as amostras foram expostas à temperatura ambiente por duas horas e, em seguida, pesadas e homogeneizadas em multiprocessador doméstico.

Para as avaliações de desempenho produtivo, o delineamento experimental foi o inteiramente ao acaso, constituído por cinco tratamentos com seis repetições de 36 aves cada. Para as análises de composição de carcaça, composição de extrato etéreo e avaliação macroscópica e microscópica do fígado, o delineamento foi o mesmo - cinco tratamentos e seis repetições cada -, sendo cada ave ou fígado considerado como uma repetição. O fígado foi avaliado macroscopicamente, considerando-se tamanho, coloração e consistência. De cada fígado, seis por tratamento, retirou-se uma amostra do lobo direito, que foi fixada em formol neutro $10 \%$, processada 
rotineiramente e corada pela hematoxilinaeosina, para análise em microscópio de luz comum.

As respostas foram regredidas em relação à suplementação de colina em seus componentes lineares e quadráticos, para escolha do modelo de regressão que melhor descrevesse as observações. Para as avaliações histológicas do fígado e para a viabilidade das aves que violaram os princípios da normalidade e homocedasticidade, o teste utilizado foi o de Kruskal-Wallis (Sampaio, 2002).

\section{RESULTADOS E DISCUSSÃO}

Os resultados obtidos para peso corporal, ganho de peso, consumo de ração, conversão alimentar e viabilidade dos frangos de corte, na fase inicial de criação, encontram-se na Tab. 2.

Tabela 2. Peso corporal (PC), ganho de peso (GP), consumo de ração (CR), conversão alimentar (CA) e viabilidade (VIA) de frangos de corte, na fase inicial de criação, de acordo com a suplementação de colina

\begin{tabular}{cccccc}
\hline Colina $(\mathrm{mg} / \mathrm{kg})$ & PC $(\mathrm{g})$ & GP $(\mathrm{g})$ & CR $(\mathrm{g})$ & CA $(\mathrm{g} / \mathrm{g})$ & VIA $(\%)$ \\
\hline zero & 975,3 & 932,1 & $1.310,8$ & 1,406 & 99,1 \\
100 & 968,4 & 925,1 & $1.293,6$ & 1,398 & 99,1 \\
200 & 977,4 & 934,5 & $1.298,2$ & 1,389 & 99,1 \\
300 & 988,7 & 945,6 & $1.313,1$ & 1,389 & 98,6 \\
400 & 961,3 & 918,0 & $1.258,4$ & 1,370 & 98,1 \\
\hline Colina $(\mathrm{mg} / \mathrm{kg})$ & $\mathrm{ns}$ & $\mathrm{ns}$ & $\mathrm{ns}$ & $\mathrm{L}$ & $*$ \\
CV $(\%)$ & 2,71 & 2,80 & 3,90 & 1,55 & 2,01 \\
\hline
\end{tabular}

ns - efeito não significativo pelo teste $\mathrm{F}(\mathrm{P}>0,05)$.

$\mathrm{L}$ - efeito linear da suplementação de colina pelo teste $\mathrm{F}(\mathrm{P} \leq 0,01)$.

* - efeito não significativo pelo teste de Kruskal-Wallis $(\mathrm{P}>0,05)$.

A suplementação de colina nas dietas não teve efeitos $(\mathrm{P}>0,05)$ sobre peso corporal, ganho de peso, consumo de ração e viabilidade das aves. Porém, houve efeito linear da suplementação de colina $(\mathrm{P} \leq 0,01)$ sobre a conversão alimentar, sendo o melhor resultado obtido quando os pintos foram alimentados com a dieta suplementada com $400 \mathrm{mg} / \mathrm{kg}$, o que correspondeu a $1.767 \mathrm{mg}$ de colina/kg de ração, de acordo com a equação de regressão: $\mathrm{Y}=$ 1,4066 - 0,00008112 X ( $\left.R^{2}=89,43\right)$, em que $X$ representa o valor da suplementação de colina e Y a conversão alimentar (Fig. 1).

O valor máximo de suplementação de colina utilizado neste experimento, 400mg/kg, está acima das recomendações de Rostagno et al. (2011), que indicaram 370 e $330 \mathrm{mg}$ de colina suplementar por kg de ração para frangos de um a sete dias e de oito a 21 dias de idade, respectivamente. Para recomendar a suplementação ótima de colina que resulte na melhor conversão alimentar, valores de suplementação acima dos avaliados devem ser testados a fim de possibilitar o ajuste de um modelo de regressão que permita estimar a melhor resposta à suplementação.

Os resultados encontrados, com exceção da conversão alimentar, estão de acordo com Carrato (1985), que, ao estudar os efeitos da substituição da metionina suplementar $(0,06 \%)$ pela combinação de diferentes valores de suplementação de cloreto de colina $(0,06 ; 0,03$; $0,015 \%)$ e sulfato de sódio $(0,10 ; 0,20 ; 0,30 \%)$, em dietas para frangos de corte, não observou benefícios nos resultados de desempenho das aves no período de um a 21 dias de idade. O mesmo fato foi observado por Sakomura et al. (1996), que realizaram um experimento em que constataram que os valores de colina contidos na dieta basal $(1.335 \mathrm{mg} / \mathrm{kg})$ foram suficientes para atender às exigências para as funções específicas da colina no organismo de frangos de corte, no período de um a 21 dias de idade. 


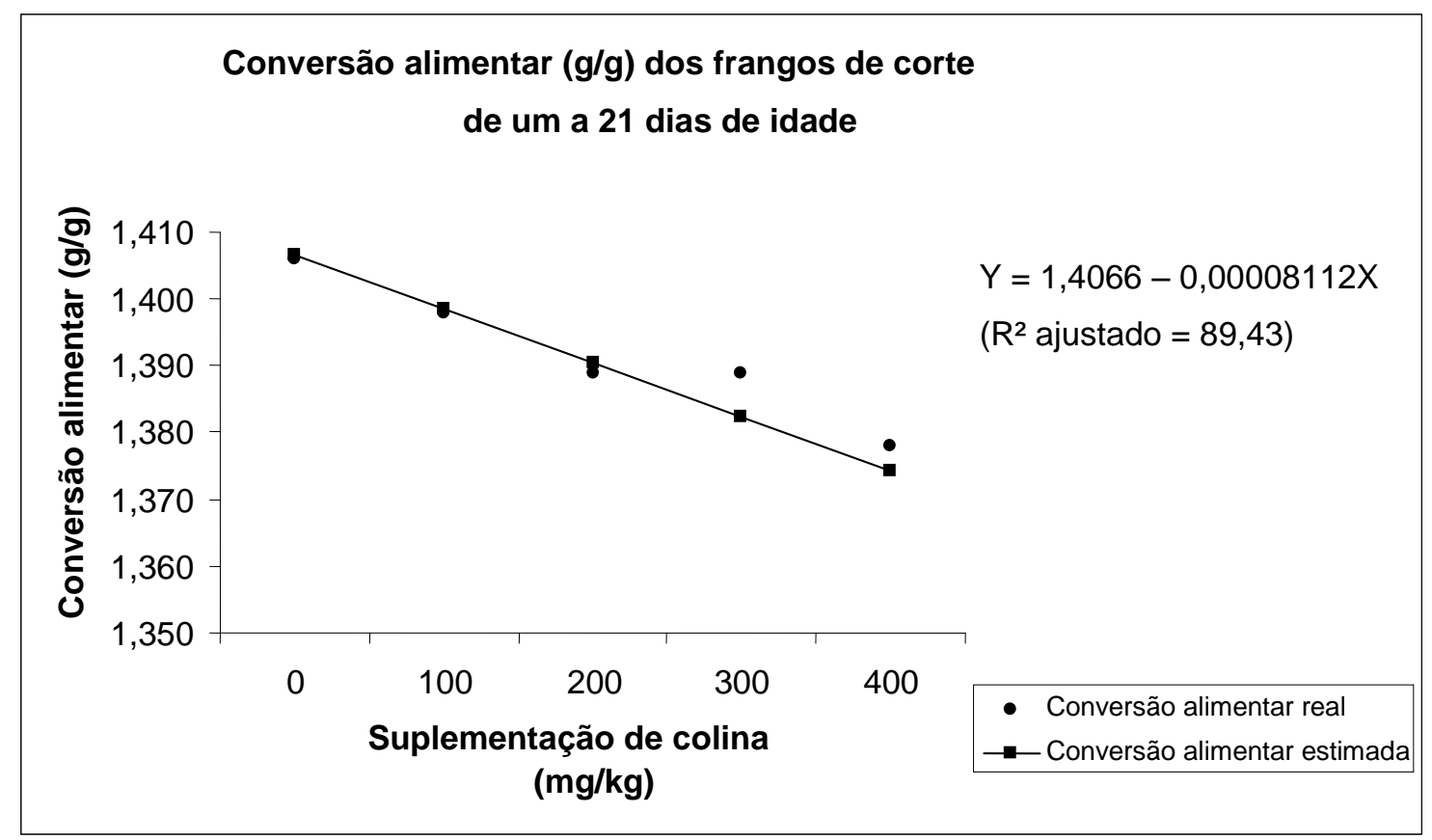

Figura 1. Conversão alimentar de frangos de corte de um a 21 dias de idade, de acordo com a suplementação de colina

De acordo com Klasing (1998), a exigência de colina será maior quanto mais elevado for o valor da proteína da dieta, a qual se deve à maior necessidade de grupamentos metil para a síntese e excreção de ácido úrico. A exigência pode ser reduzida por excesso de qualquer um dos doadores de grupos metil, como a metionina ou a betaína. Entretanto, Griffith et al. (1969) não encontraram diferenças na exigência de colina quando as aves foram alimentadas com dietas produzidas com alta e baixa proteína e suplementação de colina. Estes foram de $850 \mathrm{mg} / \mathrm{kg}$ de colina ou da combinação de colina $(850 \mathrm{mg} / \mathrm{kg})$, metionina $(0,1 \%)$ e vitamina $\mathrm{B}_{12}$ $(0,005 \mathrm{mg} / \mathrm{kg})$.

Os dados de composição de matéria seca, matéria mineral, proteína bruta e extrato etéreo, na base da matéria seca, das carcaças de frangos de corte aos 21 dias de idade estão indicados na Tab. 2.

Tabela 3. Composição percentual da carcaça de frangos de corte aos 21 dias de idade, em matéria seca (MS), matéria mineral (MM), proteína bruta (PB) e extrato etéreo (EE), na base da matéria seca, de acordo com a suplementação de colina

\begin{tabular}{ccccc}
\hline Colina $(\mathrm{mg} / \mathrm{kg})$ & MS $(\%)$ & MM $(\%)$ & PB (\%) & EE (\%) \\
\hline zero & 29,70 & 6,26 & 51,56 & 36,41 \\
100 & 30,59 & 6,43 & 50,62 & 37,11 \\
200 & 29,65 & 6,26 & 52,50 & 35,81 \\
300 & 29,83 & 6,50 & 51,17 & 36,12 \\
400 & 29,82 & 5,97 & 51,99 & 35,13 \\
\hline Colina $(\mathrm{mg} / \mathrm{kg})$ & $\mathrm{ns}$ & $\mathrm{ns}$ & $\mathrm{ns}$ & $\mathrm{ns}$ \\
CV $(\%)$ & 3,59 & 11,41 & 6,25 & 7,10 \\
\hline
\end{tabular}

ns - efeito não significativo pelo teste $\mathrm{F}(\mathrm{P}>0,05)$.

A suplementação de colina não influenciou a composição da carcaça aos 21 dias de idade das aves. Segundo Larbier e Leclercq (1994), a ausência de colina torna frágeis os fosfolipídios de membrana, o que impede a transferência de triglicerídios e colesterol para a corrente sanguínea, acumulando-os no fígado. Tal problema é causado por falta de mobilização das gorduras hepáticas. 
Os valores do extrato etéreo do fígado em função da suplementação de zero, 100, 200, 300 e 400mg/kg de colina, foram: 19,4, 18,1, 19,8, $20,9,17,7 \%$, respectivamente. Não houve efeito $(\mathrm{P}>0,05)$ da suplementação de colina sobre a composição do extrato etéreo do fígado. Essa resposta difere da encontrada por Rama Rao et al. (2001). que relataram redução significativa no conteúdo de gordura do fígado de aves que receberam dieta composta por quirera de arroz, dispondo de $1.120 \mathrm{mg} / \mathrm{kg}$ de colina, e suplementada com $760 \mathrm{mg} / \mathrm{kg}$.

Os dados de características macroscópicas e histopatológicas do fígado de frangos de corte aos 21 dias de idade estão apresentados na Tab. 4.

Tabela 4. Características macroscópicas e microscópicas do fígado de frangos de corte aos 21 dias de idade, de acordo com a suplementação de colina

\begin{tabular}{ccc}
\hline Colina $(\mathrm{mg} / \mathrm{kg})$ & Macro $^{1}$ & Histo $^{2}$ \\
\hline zero & 2 & 2 \\
100 & 2 & 3,5 \\
200 & 2 & 2 \\
300 & 2 & 3,5 \\
400 & 2 & 1 \\
\hline
\end{tabular}

${ }^{\mathrm{I}}$ Macro $=$ macroscópicas $=$ escores de coloração do fígado: normal $=1 ;$ levemente amarelado $=2$; moderadamente amarelado $=3$; intensamente amarelado $=4$.

${ }^{2}$ Histo $=$ histológicas $=$ escores de degeneração gordurosa: normal $=1 ;$ multifocal leve $=2$; multifocal moderada $=$ 3; multifocal acentuada $=4$.

Valores da mediana não diferiram entre si pelo teste Kruskal-Wallis $(\mathrm{P}>0,05)$.

Nas avaliações de coloração, todos os fígados apresentaram-se com o mesmo escore levemente amarelado -, independentemente do tratamento, portanto semelhantes entre si pelo teste de Kruskal-Wallis $(\mathrm{P}>0,05)$. Nas avaliações histopatológicas, os fígados enquadraram-se da seguinte maneira: tratamento com zero e $200 \mathrm{mg} / \mathrm{kg}$ de suplementação de colina degeneração multifocal leve; tratamento com 100 e $300 \mathrm{mg} / \mathrm{kg}$ - degeneração multifocal moderada a acentuada; tratamento com $400 \mathrm{mg} / \mathrm{kg}$ - sem alterações. Apesar das diferenças entre as classificações, os resultados foram considerados semelhantes entre si pelo teste de Kruskal-Wallis $(\mathrm{P}>0,05)$. Portanto, não houve efeito da suplementação de colina $(\mathrm{P}>0,05)$ sobre as características macroscópicas e histopatológicas do fígado. Este resultado foi semelhante ao relatado por Wolford e Murphy (1972), que não detectaram nenhuma alteração no conteúdo de gordura no fígado de aves que receberam dietas suplementadas com vitaminas lipotrópicas (vitamina $\mathrm{B}_{12}$, vitamina $\mathrm{E}$, colina, inositol), e por Pearce (1975), ao verificarem que a suplementação da dieta com colina ou inositol não afetou a incidência da síndrome do fígado gordo ou o metabolismo lipídico de frangos de corte.

\section{CONCLUSÕES}

A suplementação de colina tem efeito sobre o desempenho de frangos de corte, e a suplementação com $400 \mathrm{mg} / \mathrm{kg}$ na dieta é a que resulta em melhor conversão alimentar para os frangos de corte aos 21 dias de idade, correspondendo a $1.767 \mathrm{mg}$ de colina total na dieta/kg de ração.

\section{AGRADECIMENTOS}

Os autores agradecem à Fundação de Amparo à Pesquisa do Estado de Minas Gerais, (FAPEMIG) por fornecer fundos para publicação da pesquisa, e à Coordenação de Aperfeiçoamento de Pessoal de Nível Superior (CAPES), pela bolsa de estudos concedida ao primeiro autor. 


\section{REFERÊNCIAS BIBLIOGRÁFICAS}

BERTECHINI, A.G. Nutrição de monogástricos. Lavras: Ed. UFLA, 2006. 301p.

CARRATO, R.L.P. Substituição de metionina por cloreto de colina e sulfato de sódio em rações de frangos de corte. 1985. 52f.Tese (Mestrado) - Escola de Veterinária, Universidade Federal de Minas Gerais, Belo Horizonte, MG

FERREIRA, R.A.; NETO, A.R.O. Vitaminas na nutrição animal. In: NUTRIÇÃO animal - Tópicos Avançados. Itapetinga, BA: UESB, 2003. p.268.

GLICK, D. Concerning the reineckate method for the determination of choline. J. Biol. Chem., v.156, p.643-651, 1944.

GRIFFITH, M.; OLINDE, A.J.; SCHEXNAILDER, R. et al. Effect of choline, methionine and vitamin $b_{12}$ on liver fat, egg production and egg weight in hens. Poult. Sci., v.48, p.2160-2172, 1969.

JUKES, T.H. Effect of choline and other supplements on perosis. J. Nutr., v.20, p.445-457, 1940.

JUKES, T.H. The effect of certain organic compounds and other dietary supplements on perosis. J. Nutr., v.22, p.315-326, 1941.

KLASING, K.C. Comparative avian nutrition. Davis: CAB International, 1998. 350p.

LARBIER, M.; LECLERCQ, B. Nutrition and feeding of poultry. Nottingham: University Press, 1994. 305p.

LEITE, R.S.; ROCHA, J.S.R.; MICHELL, B.C. et al. Efeitos de planos nutricionais e de fontes de metionina sobre o desempenho, rendimento e composição de carcaças de frangos de corte. Arq. Bras. Med. Vet. Zootec., v.61, p.1120-1127, 2009.
NESHEIM, M.C.; NORVELL, M.J.; CEBALLOS, E. et al. The effect of choline supplementation of diets for growing pullets and laying hens. Poult. Sci., v.50, p.820-831, 1971.

PEARCE, J. The effects of choline and inositol on hepatic lipid metabolism and the incidence of the fatty liver and kidney syndrome in broilers. Br. Poult. Sci., v.16, p.565-570, 1975.

RAMA RAO, S.V.; SHYAM SUNDER, G.; REDDY, M.R. et al. Effect of supplementary choline on the performance of broiler breeders fed on different energy sources. Br. Poult. Sci., v.42, p.362-367, 2001.

ROSTAGNO, H.S.; ALBINO, L.F.T.; DONZELE, J.L. et al. Tabelas brasileiras para aves e suínos: composição de alimentos e exigências nutricionais. 2.ed. Viçosa, MG: UFV, 2005. 186p.

ROSTAGNO, H.S.; ALBINO, L.F.T.; DONZELE, J.L. et al. Tabelas brasileiras para aves e suínos: composição de alimentos e exigências nutricionais. 3.ed. Viçosa, MG: UFV, 2011. 252p.

SAKOMURA, N.K.; KIMURA, M.E.; JUNQUEIRA, O.M. et al. Utilização de betaína em rações de frangos de corte. ARS Vet., v.12, p.86-94, 1996.

SAMPAIO, I.B.M. Estatística aplicada à experimentação animal. 2.ed. Belo Horizonte: FEPMVZ, 2002. 265 p.

SILVA, D.J.; QUEIROZ, A.C. Análise de alimentos: métodos químicos e biológicos. Viçosa: UFV, 2002.

WOLFORD, J.H.; MURPHY, D. Effect of diet on fatty liver-hemorrhagic syndrome incidence in laying chickens. Poult. Sci., v. 51, p. 2087-2094, 1972. 